\title{
Root bark extracts of Juncus effusus and Paeonia suffruticosa protect salivary gland acinar cells from apoptotic cell death induced by cis-platinum (II) diammine dichloride
}

\author{
YOSHIKI MUKUDAI ${ }^{1}$, SEIJI KONDO ${ }^{1}$, SUNAO SHIOGAMA ${ }^{1}$, TOMOYUKI KOYAMA ${ }^{2}$, \\ CHUNNAN LI ${ }^{1,3}$, KAZUNAGA YAZAWA ${ }^{2}$ and SATORU SHINTANI ${ }^{1}$
}

\author{
${ }^{1}$ Department of Oral and Maxillofacial Surgery, School of Dentistry, Showa University, Ota-ku, Tokyo 145-8515; \\ ${ }^{2}$ Laboratory of Nutraceuticals and Functional Foods Science, Graduate School of Marine Science and Technology, \\ Tokyo University of Marine Science and Technology, Minato-ku, Tokyo 108-8477, Japan; \\ ${ }^{3}$ Medical College, Jinggangshan University, Ji'an, Jiangxi 343009, P.R. China
}

Received May 13, 2013; Accepted July 8, 2013

DOI: $10.3892 /$ or.2013.2744

\begin{abstract}
Cis-platinum (II) diammine dichloride (CDDP) is a platinum-based anticancer agent, and is often used for chemotherapy for malignant tumors, albeit CDDP has serious side-effects, including xerostomia (dry mouth). Since patients with xerostomia have reduced quality of life, it is urgent and important to identify nontoxic and natural agents capable of reducing the adverse effect of chemotherapy on salivary gland function. Therefore, we commenced an institutional collaborative project in which candidates of herbal extracts were selected from more than 400 bioactive herbal products for their potential therapeutic effects not only on xerostomia, but also on oral diseases. In the present study, we report on two Chinese medical herbal extracts from the root barks of Juncus effusus and Paeonia suffruticosa. The two extracts showed a protective effect in NS-SV-Ac cells from the cytotoxicity and apoptosis caused by CDDP. The effect was dependent on the p53 pathway, protein kinase B/ Akt 1 and mitochondrial apoptosis-related proteins (i.e. Bcl-2 and Bax), but was not dependent on nuclear factor $\kappa \mathrm{B}$. Notably, the apoptosis-protective effect of the extracts was not observed in adenocystic carcinoma cell lines. Although these extracts have been utilized in traditional Chinese medicine for hundreds of years, there are no reports to our knowledge, on their therapeutic effects on xerostomia. Thus, in the present study, we elucidated the potency of these herbal extracts as novel candidates for xerostomia to improve the quality of life of patients undergoing chemotherapy.
\end{abstract}

Correspondence to: Dr Yoshiki Mukudai, Department of Oral and Maxillofacial Surgery, School of Dentistry, Showa University, 2-1-1 Kitasenzoku, Ota-ku, Tokyo 145-8515, Japan

E-mail: mukudai@dent.showa-u.ac.jp

Key words: herbal extract, Chinese medicine, salivary gland, cis-platinum (II) diammine dichloride, xerostomia

\section{Introduction}

Oral cancer is a life-threatening disease causing $~ 500,000$ deaths annually worldwide $(1,2)$. Chemotherapy is commonly used to treat a variety of oral cancers, generally in combination with surgery or radiation (3). One of the chemotherapeutic agents often used is cis-platinum (II) diammine dichloride (CDDP), a platinum-based compound. However, like most anticancer agents, CDDP has potentially severe adverse effects such as nephrotoxicity (4), neurotoxicity (5), nausea and vomiting (6) and ototoxicity (7). One of its adverse effects in the head and neck region is xerostomia (dry mouth) $(8,9)$.

Xerostomia results from hypofunction of the salivary glands and can be caused by several physiological and iatrogenic factors, autoimmune disorders and infection $(10,11)$. Although the mechanism and therapeutics of the disease are yet to be fully elucidated, salivary gland dysfunction can lead to impaired speech, loss of taste perception and appetite, and infection, thereby reducing quality of life. Hence most physicians and basic scientists recognize this disorder as an important issue for head and neck cancer patients undergoing chemotherapy.

Traditional Chinese medicine has been used for a variety of diseases for several thousands of years. With this in mind, an inter-institutional collaborative project involving Showa University and Tokyo University of Marine Science and Technology was launched in 2010, in order to elucidate herbal extracts as potential therapeutic candidates for disorders of the head-and-neck region (12). The project examined more than 400 bioactive herbal products. After a preliminary experiment, in which herbal extracts were examined for prevention of salivary gland acinar cell death, the root bark of Juncus effusus (J.effusus, commonly known as soft rush) and Paeonia suffruticosa (P. suffruticosa, commonly known as Chinese tree peony) were focused on.

The present in vitro study demonstrated that the methanolic extracts of these herbs are capable of preventing apoptotic cell death from CDDP in salivary gland acinar cells, but not in carcinoma cells. The findings suggest that these herbal extracts 
may have potential as novel therapeutic agents for xerostomia, and may accordingly improve quality of life during chemotherapy for head and neck cancer patients.

\section{Materials and methods}

Preparation of the root bark. J. effusus and P. suffruticosa were cultivated in China, and the herbal extracts were prepared in that country before being imported to Japan. A specimen of each was deposited in the herbarium of the Tokyo University of Marine Science and Technology. Dry powdered roots (100 g) were extracted by distilled water and concentrated to $1 \mathrm{mg} / \mathrm{ml}$ under reduced pressure.

Cell culture. An immortalized human salivary gland acinar cell line (NS-SV-Ac), a kind gift from Dr Masayuki Azuma (University of Tokushima, Japan), was cultured as described elsewhere (13). Acc 2 and Acc M (human adenocystic carcinoma cell lines) were cultured as described previously (14). All cells were grown at $37^{\circ} \mathrm{C}$ in an atmosphere containing $5 \% \mathrm{CO}_{2}$ and $100 \%$ humidity.

Histochemistry. The cells were seeded at a density of $3 \times 10^{3}$ cells/well in 48 -well cell culture plates. The next day, $20 \mu \mathrm{g} / \mathrm{ml}$ of CDDP (Nippon Kayaku, Tokyo, Japan) with or without the herbal extracts (at concentrations of 1 and $10 \mu \mathrm{g} / \mathrm{ml}$ adjusted by phosphate-buffered saline) were added to the medium. After 3 days, the cells were fixed and stained with crystal violet or toluidine blue, as described previously (15).

Cell viability and apoptosis assays. For the 3-[4,5-dimethylthiazol-2-yl]-2,5-diphenyltetrazolium bromide (MTT) assay, the cells were seeded at a density of $1 \times 10^{3}$ cells/well in 96-well cell culture plates. They were treated as described above, and the MTT assay was performed as described previously (16). The activities of caspase 3/7, 8 and 9 were measured using the Caspase-Glo Assay and GloMax-Multi Plus Detection System (both from Promega Corporation, Madison, WI, USA), according to the manufacturer's protocol. Genomic DNA fragmentation was investigated using a DNA ladder assay; this was performed with a commercial kit (ApopLadder EX; Takara, Shiga, Japan), according to the manufacturer's protocol.

Western blot analysis. Total cellular protein was prepared as described previously (17), and the protein concentration was measured with Quick Start Bradford reagent (Bio-Rad, Hercules, CA, USA) using bovine serum albumin as a standard, and aliquots were stored at $-80^{\circ} \mathrm{C}$ until use. Protein samples $(20 \mu \mathrm{g})$ were subjected to sodium dodecyl sulfate-polyacrylamide electrophoresis (SDS-PAGE) in 4-20\% gradient gel (Bio-Rad), and the blots were transferred onto a polyvinylidene difluoride membrane (Life Technologies, Carlsbad, CA, USA). The blots were blocked, incubated with primary and horseradish peroxidase-conjugated secondary antibodies, and washed as previously described (17). Subsequently, the signal was visualized using Amersham ECL western blotting detection reagents (GE Healthcare, UK Ltd., Buckinghamshire, UK) and the ChimiDoc XRS Plus ImageLab System (Bio-Rad). The primary antibodies were purchased from Cell Signaling Technology, Inc. (Danvers, MA, USA) and Santa Cruz
Biotechnology, Inc. (Santa Cruz, CA, USA), and secondary antibodies were purchased from GE Healthcare.

Dual luciferase assay (DLA). Three firefly luciferase reporter vectors (pGL4.24, pGL4.32 and pGL4.38) were purchased from Promega Corporation. pGL4.32 and pGL4.38 contain the human $\kappa \mathrm{B}$ element and $\mathrm{p} 53$ response element respectively, in a promoter legion of the firefly luciferase gene, while pGL4.24 has only a minimal promoter (minP) in the corresponding region. pRL-TK (Promega Corporation) was used as an internal control for transfection, as described previously (18). We seeded 5,000 cells in a 96-well culture plate, and the next day, the cells were transfected with $200 \mathrm{ng}$ of pGL4.24, pGL4.32, or pGL4.38 plus 10 ng of pRL-TK using Lipofectamine 2000 (Life Technologies), and cultured as described above. After 3 days, the DLA was performed with the Dual-Luciferase Reporter Assay System and GloMaxMulti Detection System (both from Promega Corporation), according to the manufacturer's protocol.

Statistical analysis. Unless otherwise specified, all experiments were repeated at least 3 times and similar results were obtained in the repeated experiments. Statistical analysis was performed using repeated measure analysis of paired Student's t-tests. Data are expressed as means \pm standard deviation of triplicate data.

\section{Results}

Extracts of J. effusus and P. suffruticosa protect NS-SV-Ac cells from cytotoxicity induced by $C D D P$. As described in our recent study (12), more than 400 bioactive herbal extracts were subjected to in vitro preliminary screening in which NS-SV-Ac cells were cultured for 3 days in the presence of the extracts plus $20 \mu \mathrm{g} / \mathrm{ml}$ of CDDP (data not shown). That study identified 2 herbal extracts, J. effusus and P. suffruticosa, as being able to protect cells from CDDP-induced cell death, at a minimum concentration of $1 \mu \mathrm{g} / \mathrm{ml}$ (Fig. 1A). The present MTT assay reinforced the histochemical results by showing that $20 \mu \mathrm{g} / \mathrm{ml}$ of CDDP was sufficiently cytotoxic to induce cell death in NS-SV-Ac cells, but that cell viability was protected in the presence of 1 or $10 \mu \mathrm{g} / \mathrm{ml}$ of either extract (Fig. 1B).

Herbal extracts prevent NS-SV-Accells from apoptosis induced by $C D D P$. It is well known that CDDP inhibits the synthesis of DNA and induces apoptotic cell death (4-9). The DNA ladder assay showed that the genomic DNA of NS-SV-Ac cells in the CDDP-containing medium was fragmented, and indicated that the cell death was caused by apoptosis (Fig. 2A). However, addition of the herbal extracts reduced this fragmentation in a dose-dependent manner (Fig. 2A). Similarly, although the activities of caspase 3/7, 8 and 9 were increased by CDDP, addition of the herbal extracts reduced all these activities to basal levels, even at the concentration of $1 \mu \mathrm{g} / \mathrm{ml}$ (Fig. 2B-D). The results suggest that the herbal extracts have the pharmacological capability to protect normal salivary gland acinar cells from apoptotic cell death induced by CDDP.

Herbal extracts prevent apoptosis by upregulating Bcl-2 and $B c l-X_{L}$ via activation of the Akt pathway. Next, we investigated 
A
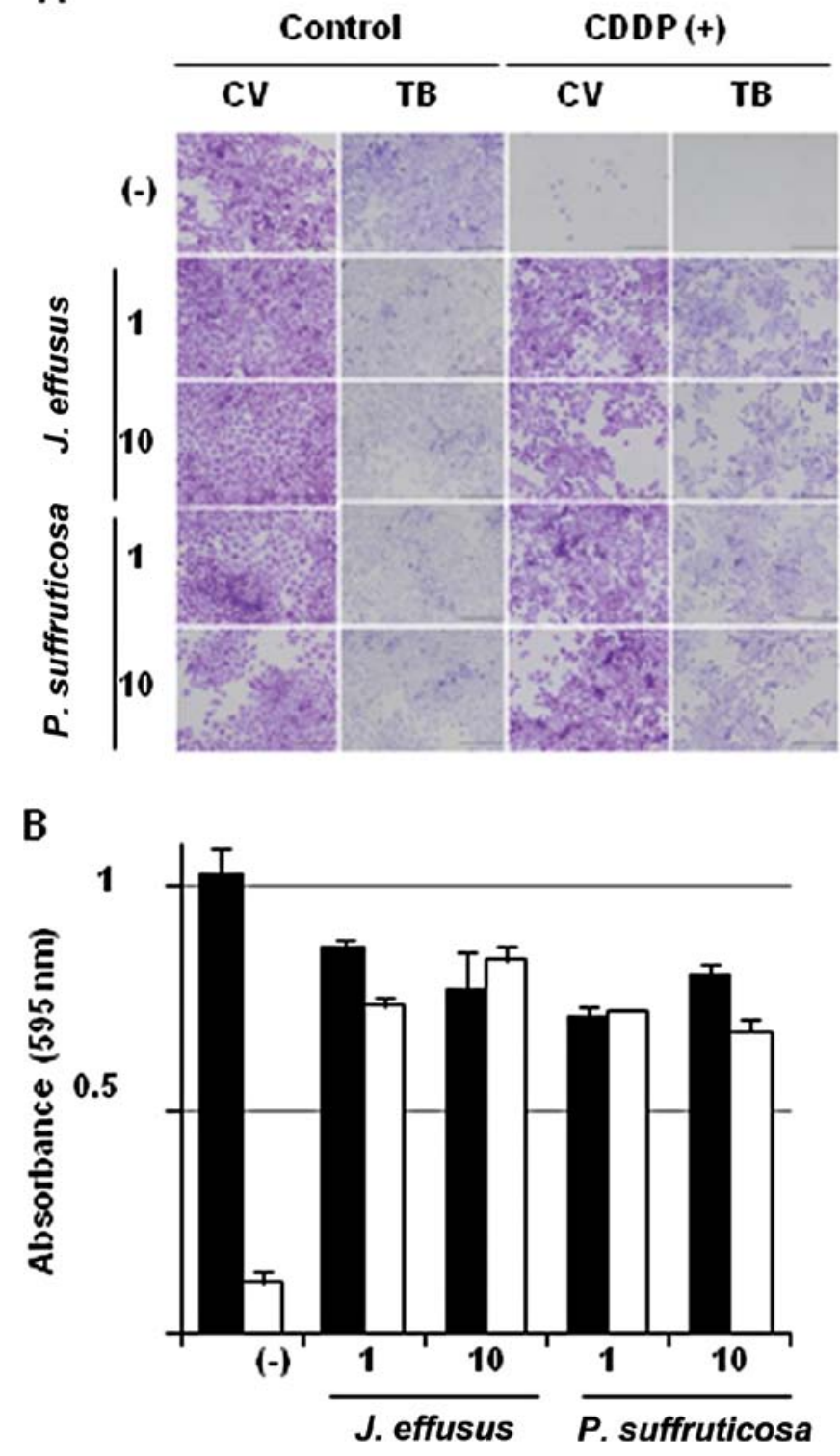

Figure 1. Herbal extracts protect salivary gland cells against cell death induced by CDDP. (A) NS-SV-Ac cells were grown in the absence (-) or presence of 1 or $10 \mu \mathrm{g} / \mathrm{ml}$ of the extracts of J. effusus or P. suffruticosa for 3 days, and the medium was then replaced with fresh control or CDDP-containing medium [CDDP (+)]. After $24 \mathrm{~h}$, the cells were stained using crystal violet (CV) and toluidine blue (TB). Bar, $100 \mu \mathrm{m}$. (B) Cells were grown as described above, and the medium was replaced with fresh control (closed box) or CDDP-containing medium (open box). Thereafter, an MTT assay was performed. Data are means \pm standard deviations of 3 cultures. CDDP, cis-platinum (II) diammine dichloride.

the protein(s) involved in the preventive effects of the herbal extracts on apoptosis. Accordingly, western blotting for various apoptosis-related proteins was performed (Fig. 3A). Addition of CDDP resulted in cleavage of poly(ADP-ribose) polymerase (PARP) 1; however, this cleavage was almost completely inhibited by the herbal extracts, regardless of an increase in p53 protein concentration. Regarding mitochondrial apoptosisrelated proteins, expressions of the pro-apoptotic proteins Bax, Bad, Bid and Bak were very similar among the samples. However, it is noteworthy that addition of the herbal extracts significantly increased the expression of $\mathrm{Bcl}-2$ and $\mathrm{Bcl}-\mathrm{X}_{\mathrm{L}}$
A
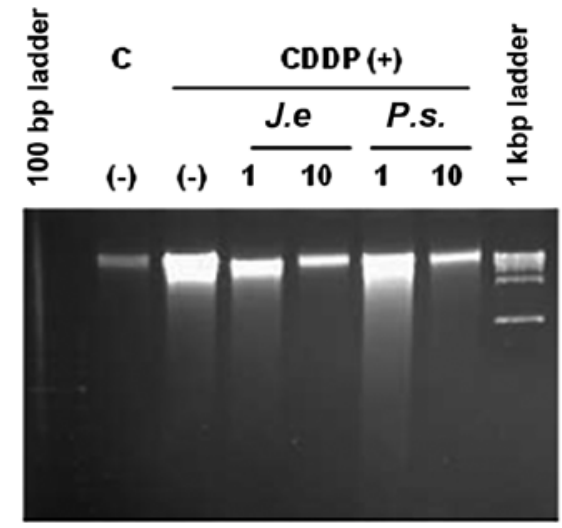

B
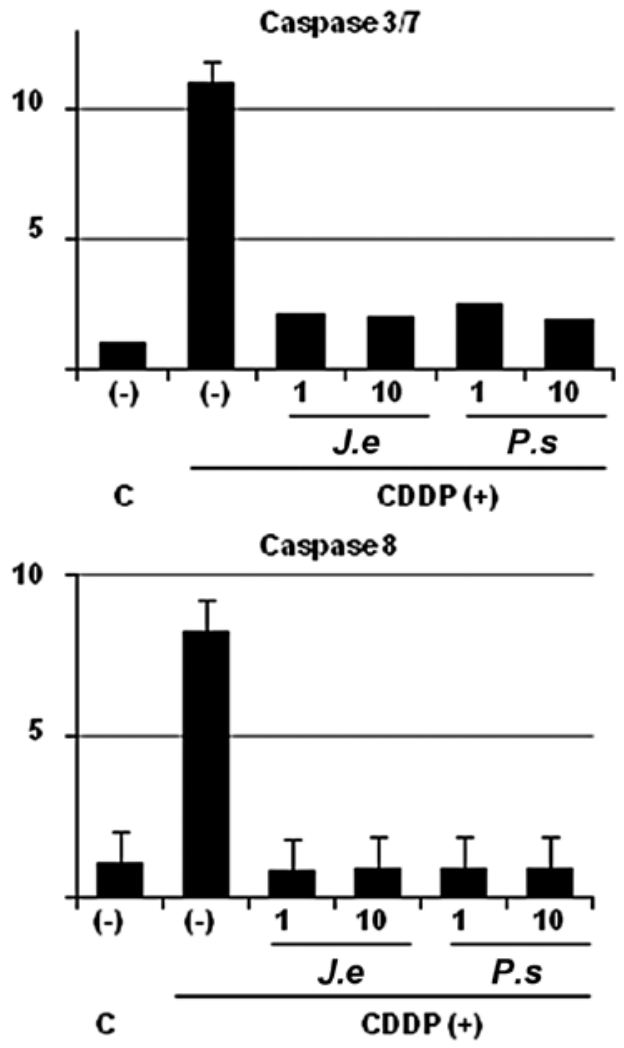

D

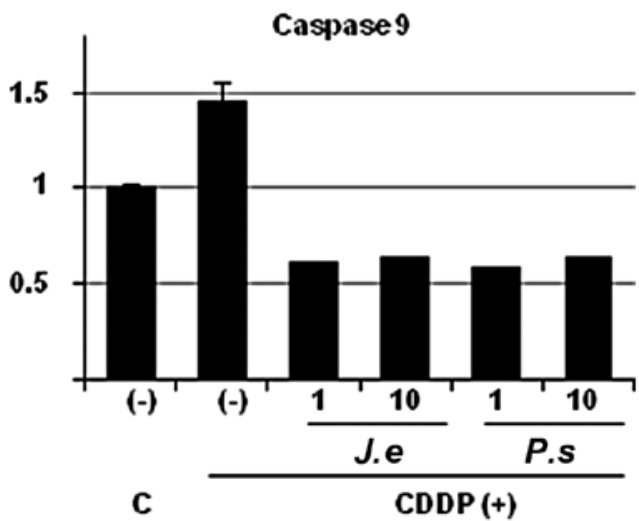

Figure 2. Herbal extracts prevent salivary gland cells from apoptotic cell death induced by CDDP. NS-SV-Ac cells were grown in the absence (-) or presence of 1 or $10 \mu \mathrm{g} / \mathrm{ml}$ of J. effusus (J.e) or P. suffruticosa (P. s) for 3 days, and the medium was then replaced with fresh control (C) or CDDPcontaining medium [CDDP (+)]. After $24 \mathrm{~h}$, the cells were harvested and subjected to (A) DNA ladder assay with 100-bp and 1-kbp ladder markers shown at the right and left side of adjacent lanes, respectively. The cells were also subjected to (B) caspase 3/7, (C) caspase 8 and (D) caspase 9 activity assays. CDDP, cis-platinum (II) diammine dichloride. 
A

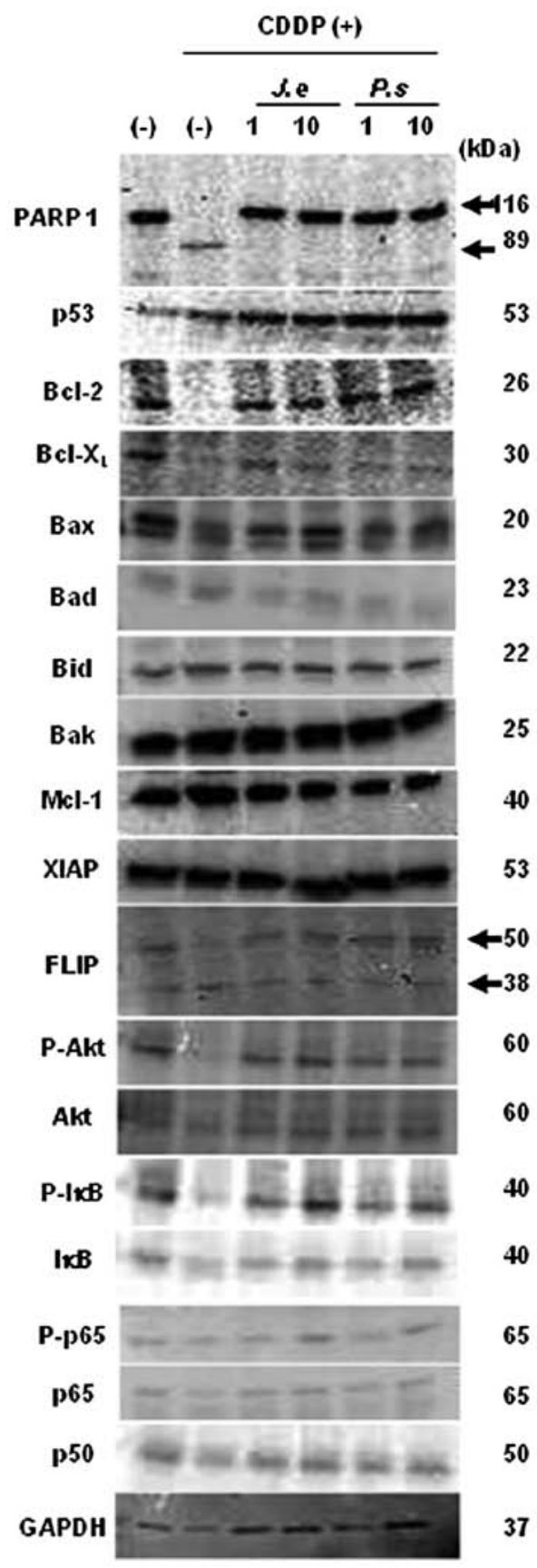

B
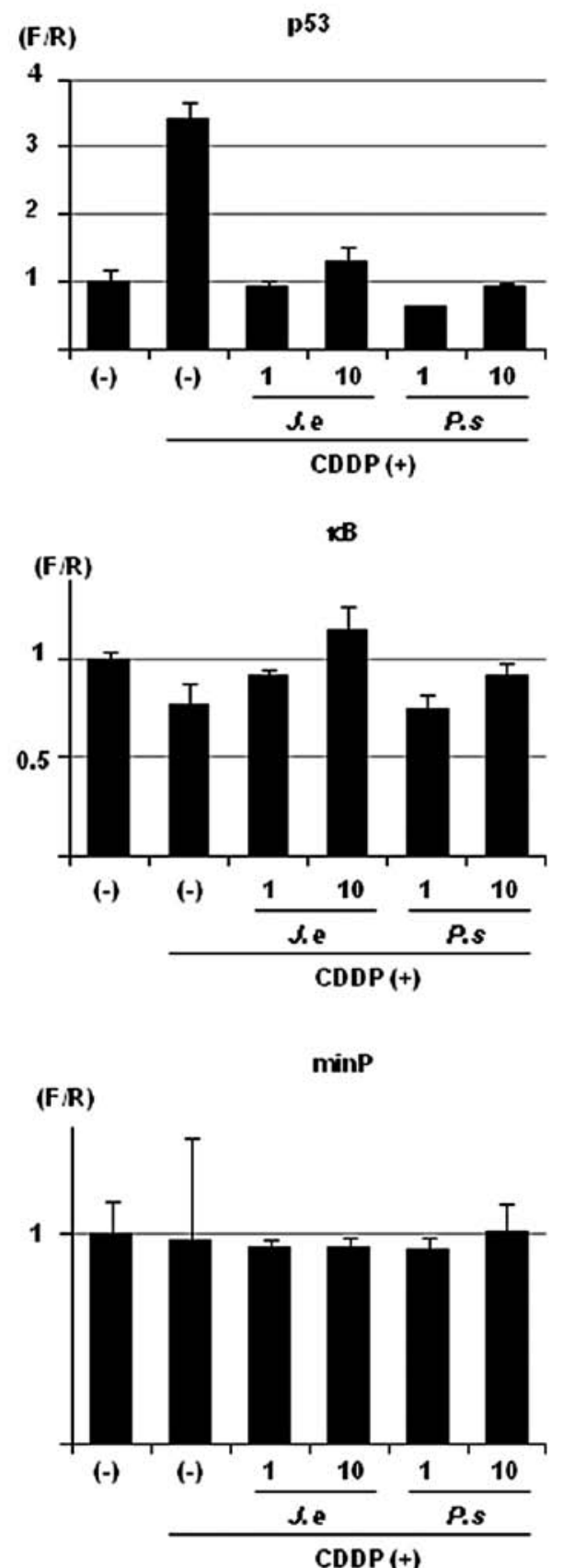

Figure 3. Herbal extracts prevent salivary gland cells from p53-, Bcl-2/Bax- and Akt 1-mediated apoptosis induced by CDDP. (A) NS-SV-Ac cells were grown in the absence (-) or presence of 1 or $10 \mu \mathrm{g} / \mathrm{ml}$ of J. effusus (J.e) or P. suffruticosa (P. s) for 3 days, and the medium was then replaced with fresh control or CDDP-containing medium [CDDP (+)]. After $12 \mathrm{~h}$, total cellular protein was purified and subjected to western blotting for 19 apoptosis-related proteins [PARP 1, p53, Bcl-2, Bcl- $\mathrm{L}_{\mathrm{L}}$, Bax, Bad, Bid, Bak, Mcl-1, XIAP, FLIP, phosphorylated (P)-Akt, Akt, P-IкB, IкB, P-p65, p65 and p50] as well as for GAPDH as an internal control. The molecular weights of interest are shown at the right adjacent side of the panel in $\mathrm{kDa}$. (B) The cells were treated as described above, and were then subjected to a dual luciferase assay for the $\mathrm{p} 53$ response element and $\kappa \mathrm{B}$ element, and for a minimal promoter (minP) as a negative control. CDDP, cis-platinum (II) diammine dichloride.

(pro-survival proteins). Expression of other apoptosis-related proteins was modulated only slightly, if at all, by the extracts (e.g., FLIP showed minor upregulation, whereas expression of Mcl-1 and XIAP remained very similar). Furthermore, although phosphorylation of protein kinase B/Akt 1 (Akt 1), which plays a crucial role in cell survival, was barely observed after addition of CDDP, this was prevented by addition of the extracts. In contrast, phosphorylation of nuclear factor- $\mathrm{kB}$ (NF-kB)-related proteins was slightly modulated by the herbal extracts. Next, we used DLA to investigate the involvement of promoter activity of the p53 response element and $\kappa \mathrm{B}$ element (Fig. 3B). CDDP increased p53 response element activity, and this activation was abolished by the extracts. Meanwhile, $\kappa B$ element activity was not modulated by CDDP, although it was increased slightly by the extracts. The reporter activity of pGL4.24, a negative control, was not altered, regardless of the presence or absence of CDDP or the extracts. Therefore, the results suggest that the reporter activity depends 
A

Acc 2

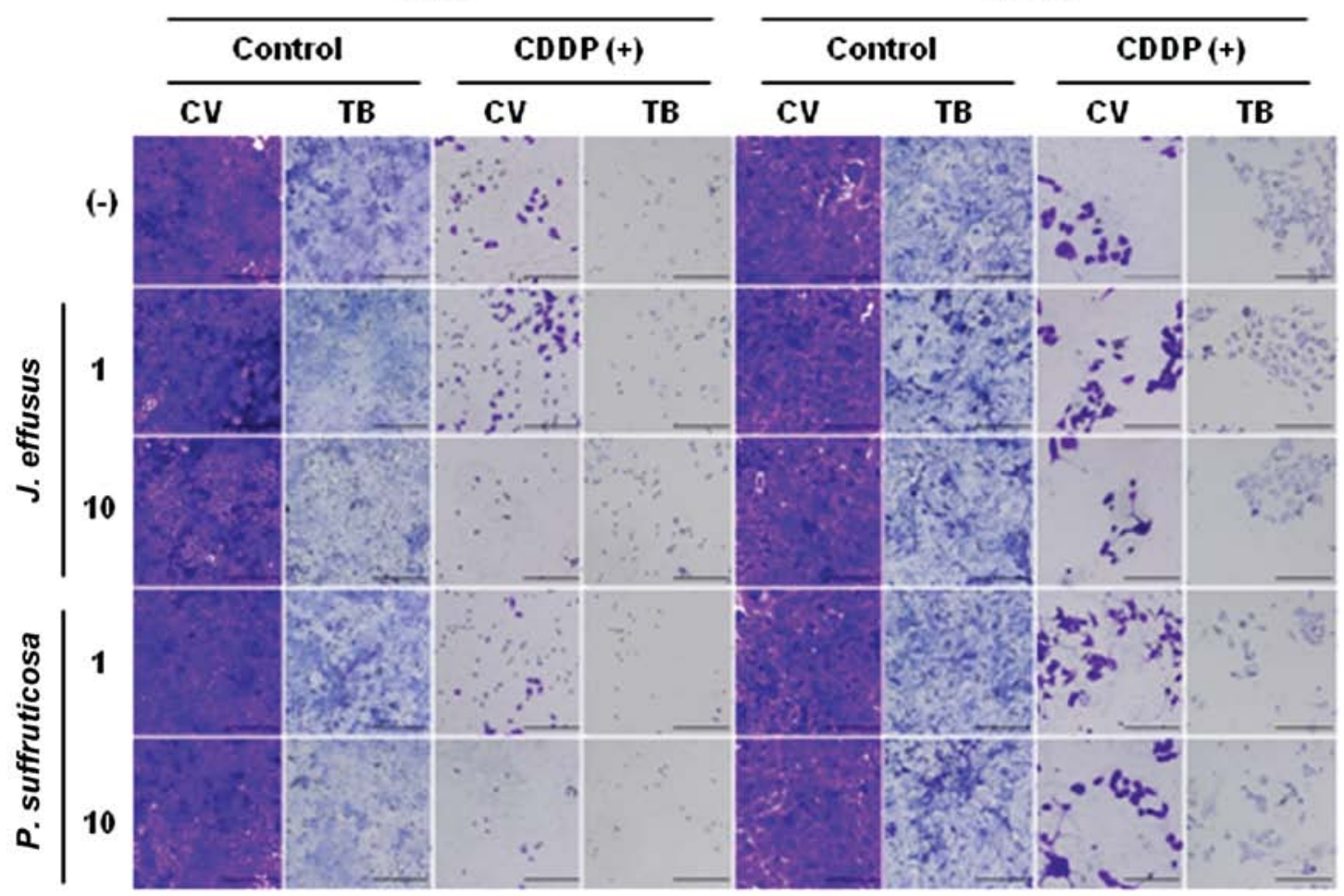

B

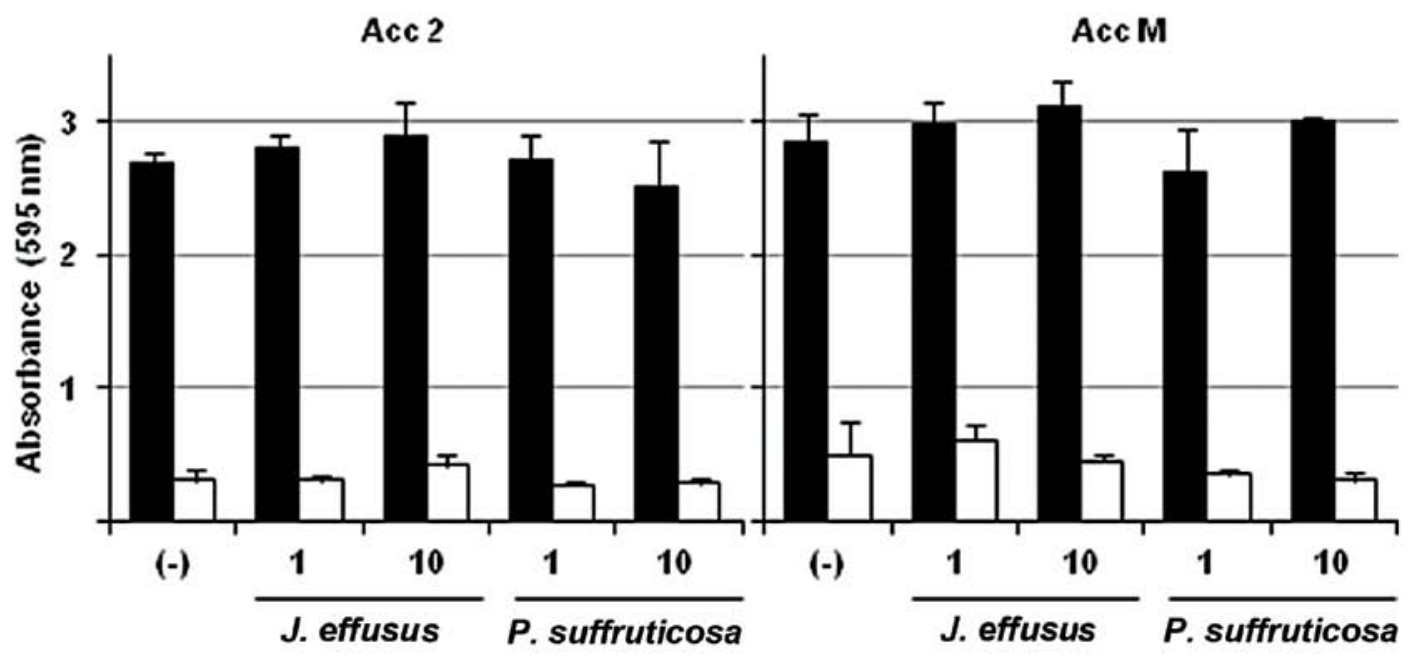

Figure 4. Herbal extracts exhibit no preventive effect against cell death induced by CDDP in adenocystic carcinoma cells. (A) Acc 2 and Acc M cells were grown in the absence (-) or presence of 1 or $10 \mu \mathrm{g} / \mathrm{ml}$ of the extracts of $J$. effusus and P. suffruticosa for 3 days, and the medium was then replaced with fresh control or CDDP-containing medium [CDDP (+)]. After $24 \mathrm{~h}$, the cells were fixed, and stained using crystal violet (CV) and toluidine blue (TB). Bar, $100 \mu \mathrm{m}$. (B) The cells were grown as described above, and the medium was replaced with fresh control (closed boxes) or CDDP-containing medium (open boxes). Thereafter an MTT assay was performed. Data are means \pm standard deviations of 3 cultures.

on the promoter sequence of each vector. Taken together, these findings suggest that CDDP decreases the phosphorylation of Akt 1 and induces apoptosis through the p53 and mitochondrial apoptotic pathways, not through $\mathrm{NF}-\kappa \mathrm{B}$. The results also suggest that the herbal extracts are capable of interrupting the p53 and mitochondrial apoptotic pathways, thereby preventing apoptosis.

The herbal extracts show no apoptosis-preventive effects in adenocystic carcinoma cell lines. In our pilot study (data not shown), several herbal extracts showed a proliferative effect not only in normal cells, but also in malignant cell lines. The magnitude of this effect varied among the herbs tested. We therefore investigated whether the extracts of J. effusus and $P$. suffruticosa exhibit anti-apoptotic or proliferative effects in malignant cells originating from salivary gland acini. In this experiment, we utilized 2 adenocystic carcinoma cell lines, Acc 2 and Acc M. As shown in Fig. 4A, in these cell lines CDDP induced marked cell death, which was probably apoptotic. Of note, the herbal extracts showed no protective 
effect on this cell death, even at the concentration of $10 \mu \mathrm{g} / \mathrm{ml}$. The MTT assay (Fig. 4B) showed the same result as observed upon histochemistry; i.e., the extracts did not inhibit the CDDP-induced apoptotic effect. These results suggest a potential advantage of the herbal extracts as therapeutic agents for xerostomia during chemotherapy for CDDP.

\section{Discussion}

Oral cancer patients usually undergo chemotherapy in combination with surgery and radiation. Since CDDP pharmacologically impairs DNA synthesis and transcription, it exerts cytotoxic and apoptotic effects not only on cancer cells but also on normal cells. As a result, the salivary glands are damaged, their function is inhibited, and patients undergoing this type of chemotherapy often develop xerostomia $(10,11)$. As chemotherapy-induced xerostomia leads to great restriction of quality of life, it is important to identify nontoxic agents capable of counteracting the effect of chemotherapy on salivary gland function. Several studies have reported that Chinese traditional herbs (19) and natural agents (20) are available for the treatment of xerostomia. Therefore, in the present institutional collaborative project, we explored herbal extracts that may have therapeutic effects not only on xerostomia, but also on oral and skeletal diseases (12).

J. effusus is a species of the Juncus plant, and traditionally is used as an anti-pyretic and anti-phlogistic agent $(21,22)$. On the other hand, P. suffruticosa is used for atherosclerosis, infection, inflammation, cutaneous disease (23) and diabetes (24), and is also reported to possess potent anti-oxidant, antimutagenic, anti-proliferative, anti-invasive, anti-arrhythmic, anti-inflammatory, anti-diabetic and anti-obesity properties (25). While previous reports on these herbs have addressed pharmacological availability, we herein revealed that these extracts are capable of preventing CDDP-induced cell death, which is mainly caused by apoptosis, in normal salivary gland cells (Figs. 1 and 2).

p53 (26), Akt 1 (27), several mitochondrial proteins (e.g., Bcl-2 and Bax) (28) and NF- $\mathrm{BB}$ (29) are well known as key regulatory molecules during apoptotic cell death. In the present study, expression of p53, Akt 1, and apoptosisrelated mitochondrial proteins was modulated during CDDP-induced apoptosis, and these changes were prevented by the extracts, while changes in NF- $\mathrm{KB}$ were not induced by CDDP (Fig. 3A and B). These finding support those of Azuma et al (30), who reported that CDDP-induced apoptosis was independent of the NF- $\mathrm{KB}$ pathway.

Finally, but most importantly, given our goal of using the extracts to treat xerostomia during chemotherapy, we investigated whether the herbal extracts had an anti-apoptotic effect on malignant cells similar to that observed in NS-SV-Ac cells. Neither histochemistry (Fig. 4A) nor MTT assay (Fig. 4B) showed that the death of malignant cells was prevented, suggesting that these extracts could have potential clinical benefit. The detailed mechanism of the cell-specific preventive effect on apoptosis remains to be further elucidated. Nonetheless, our previous study (12), in addition to previous studies examining a number of herbs, revealed that various herbal extracts have anticancer effects. Hence, one may hypothesize a switching mechanism between the triggering of and prevention of apoptosis, depending on the type of cell. In any case, further investigation, including isolation and analysis of bioactive chemicals, detailed molecular and cellular experiments in vitro, and pre-clinical studies in vivo, is of course required. Several of these studies are currently underway, and our findings will be reported in the near future.

\section{Acknowledgements}

This study was supported by Grants-in-Aid for Scientific Research (KAKENHI) (B) (to S.H.) and (C) (to Y.M. and S.K.) from the Japan Society for the Promotion of Science (JSPS), and the Nakatomi Foundation (to Y.M.). The authors wish to thank Drs Yasuto Yoshihama, Tatsuo Shirota, Hiroaki Kamatani, Yasumasa Yoshizawa, Tomohiko Kutsuna, Sayaka Yoshiba, Daisuke Soga, Daisuke Sato, Rika Nagasaki, Ryota Kishigami, and Yuji and Sayaka Kurihara for their helpful suggestions and Ms. Miho Yoshihara for the secretarial assistance.

\section{References}

1. Choi S and Myers JN: Molecular pathogenesis of oral squamous cell carcinoma: implications for therapy. J Dent Res 87: 14-32, 2008.

2. Scully C and Bagan JV: Recent advances in oral oncology 2008; squamous cell carcinoma imaging, treatment, prognostication and treatment outcomes. Oral Oncol 45: e25-e30, 2009.

3. Price KA and Cohen EE: Current treatment options for metastatic head and neck cancer. Curr Treat Options Oncol 13: 35-46, 2012.

4. dos Santos NA, Carvalho Rodrigues MA, Martins NM and dos Santos AC: Cisplatin-induced nephrotoxicity and targets of nephroprotection: an update. Arch Toxicol 86: 1233-1250, 2012.

5. McWhinney SR, Goldberg RM and McLeod HL: Platinum neurotoxicity pharmacogenetics. Mol Cancer Ther 8: 10-16, 2009.

6. Herrstedt J: Antiemetics: an update and the MASCC guidelines applied in clinical practice. Nat Clin Pract Oncol 5: 32-43, 2008.

7. Rybak LP, Mukherjea D, Jajoo S and Ramkumar V: Cisplatin ototoxicity and protection: clinical and experimental studies. Tohoku J Exp Med 219: 177-186, 2009.

8. Rapidis AD, Trichas M, Stavrinidis E, et al: Induction chemotherapy followed by concurrent chemoradiation in advanced squamous cell carcinoma of the head and neck: final results from a phase II study with docetaxel, cisplatin and 5-fluorouracil with a four-year follow-up. Oral Oncol 42: 675-684, 2006.

9. Psyrri A, Kwong M, DiStasio S, et al: Cisplatin, fluorouracil, and leucovorin induction chemotherapy followed by concurrent cisplatin chemoradiotherapy for organ preservation and cure in patients with advanced head and neck cancer: long-term follow-up. J Clin Oncol 22: 3061-3069, 2004.

10. Jensen SB, Pedersen AM, Vissink A, et al: A systematic review of salivary gland hypofunction and xerostomia induced by cancer therapies: prevalence, severity and impact on quality of life. Support Care Cancer 18: 1039-1060, 2010.

11. Napeñas JJ, Brennan MT and Fox PC: Diagnosis and treatment of xerostomia (dry mouth). Odontology 97: 76-83, 2009.

12. Li C, Yazawa K, Kondo S, et al: The root bark of Paeonia moutan is a potential anticancer agent in human oral squamous cell carcinoma cells. Anticancer Res. 32: 2625-2630, 2012.

13. Azuma M, Tamatani T, Kasai Y and Sato M: Immortalization of normal human salivary gland cells with duct-, myoepithelial-, acinar-, or squamous phenotype by transfection with SV40 orimutant deoxyribonucleic acid. Lab Invest 69: 24-42, 1993.

14. Klosek SK, Nakashiro K, Hara S, Shintani S, Hasegawa H and Hamakawa H: CD151 forms a functional complex with c-Met in human salivary gland cancer cells. Biochem Biophys Res Commun 336: 408-416, 2005.

15. Banka S, Mukudai Y, Yoshihama Y, Shirota T, Kondo S and Shintani S: A combination of chemical and mechanical stimuli enhances not only osteo- but also chondro-differentiation in adipose-derived stem cells. J Oral Biosci 54: 188-195, 2012. 
16. Tsukamoto H, Kondo S, Mukudai Y, et al: Evaluation of anticancer activities of benzo[c]phenanthridine alkaloid sanguinarine in oral squamous cell carcinoma cell line. Anticancer Res 31: 2841-2846, 2011

17. Yasuda A, Kondo S, Nagumo T, et al: Anti-tumor activity of dehydroxymethylepoxyquinomicin against human oral squamous cell carcinoma cell lines in vitro and in vivo. Oral Oncol 47: 334-339, 2011.

18. Mukudai Y, Kubota S, Eguchi T, Kondo S, Nakao K and Takigawa M: Regulation of chicken $c c n 2$ gene by interaction between RNA cis-element and putative trans-factor during differentiation of chondrocytes. J Biol Chem 280: 3166-3177, 2005.

19. Murakami M, Wei MX, Ding W and Zhang QD: Effects of Chinese herbs on salivary fluid secretion by isolated and perfused rat submandibular glands. World J Gastroenterol 15: 3908-3915, 2009.

20. Yamamoto T, Staples J, Wataha J, et al: Protective effects of EGCG on salivary gland cells treated with $\gamma$-radiation or $c i s$ platinum(II)diammine dichloride. Anticancer Res 24: 3065-3073, 2004.

21. Hanawa F, Okamoto $M$ and Towers GH: Antimicrobial DNA-binding photosensitizers from the common rush, Juncus effusus. Photochem Photobiol 76: 51-56, 2002.

22. Behery FA, Naeem ZE, Maatooq GT, et al: Phenanthrenoids from Juncus acutus L., new natural lipopolysaccharide-inducible nitric oxide synthase inhibitors. Chem Pharm Bull 55: 12641266,2007
23. Hong $\mathrm{MH}, \mathrm{Kim} \mathrm{JH}, \mathrm{Na} \mathrm{SH}$, et al: Inhibitory effects of Paeonia suffruticosa on allergic reactions by inhibiting the NF-kappaB/I kappaB-alpha signaling pathway and phosphorylation of ERK in an animal model and human mast cells. Biosci Biotechnol Biochem 74: 1152-1156, 2010.

24. Poon TY, Ong KL and Cheung BM: Review of the effects of the traditional Chinese medicine Rehmannia Six Formula on diabetes mellitus and its complications. J Diabetes 3: 184-200, 2011.

25. Choi HS, Seo HS, Kim JH, Um JY, Shin YC and Ko SG: Ethanol extract of Paeonia suffruticosa Andrews (PSE) induced AGS human gastric cancer cell apoptosis via fas-dependent apoptosis and MDM2-p53 pathways. J Biomed Sci 19: 82, 2012.

26. Meulmeester E and Jochemsen AG: p53: a guide to apoptosis. Curr Cancer Drug Targets 8: 87-97, 2008.

27. Manning BD and Cantley LC: AKT/PKB signaling: navigating downstream. Cell 129: 1261-1274, 2007.

28. Brenner D and Mak TW: Mitochondrial cell death effectors. Curr Opin Cell Biol 21: 871-877, 2009.

29. Hayden MS and Ghosh S: Shared principles in NF- $\kappa$ B signaling. Cell 132: 344-362, 2008.

30. Azuma M, Tamatani T, Ashida Y, Takashima R, Harada K and Sato M: Cisplatin induces apoptosis in oral squamous carcinoma cells by the mitochondria-mediated but not the NF- $\mathrm{\kappa B}$-suppressed pathway. Oral Oncol 39: 282-289, 2003. 